\title{
Snake Bite;Treat the Shock And Save the Kidney -with Low Dose Asv--A Practical Approach
}

\author{
Dr.T.Ravi Kumar, ${ }^{1}$ Dr.C.Manokaran ${ }^{2}$, Dr.S.Ramaswamy ${ }^{3}$. Dr.N.Kalaisezhian ${ }^{4}$ \\ Dr.P.Saravanan ${ }^{5}$, Dr.M. Gowrisankar ${ }^{6}$ Dr.Chethan Bharthwaj ${ }^{7}$, \\ Dr.Vasif Mayan $\mathrm{M} \mathrm{C}^{8}$ \\ ${ }^{1}$ Professor and Hod Dept of medicine, Govt medical college and ESIc hospital Coimbatore \\ ${ }^{2}$ Professor-Sri Manakula Vinayagar Medical College, pondicherry \\ ${ }^{3}$ Professor govt mohan kumarmangalam medical college hospital salem \\ ${ }^{4}$ Asst.Professor govt mohan kumarmangalam medical college hospital salem \\ ${ }^{5}$ Asst.Professor-Madurai Medical College Madurai \\ ${ }^{6}$ Asst.Professor-Coimbatore Medical College. post graduateGMKMCH,SALEM
}

\begin{abstract}
Morbidity and mortality due to snake bite is one of the important healthcare concerns in India especially in the rural population. Acute kidney injury which may or may not lead to chronic renal failure is one of the important risks of snake bite. We submit a study done over 3 years in the tertiary care hospital in Tamil Nadu which emphasizes on early hydration and low dose ASV as a measure for better clinical response and prevention of acute kidney injury.

Aim Of The Study: The aim of the study is to prove that low dose ASV is useful in reducing the morbidity and mortality as per the national and world health organization guide lines

Material And Methods: This is a prospective observational study in a tertiary medical care hospital ,Coimbatore in western part of Tamil Nadu, south India, between Jan 2013 to Dec 2015 with 1224 patients all are presented with history of snake bite and signs of envenomation exclusion criteria all patients with pre existing renal disease, those who are on drugs like aspirin and clopidogrel, and patients with previous history of bleeding diathesis are excluded from this study

Results: The average ASV administered was 10-15 vials much less than the prescribed maximum dose of 30 vials, even patients developed acute kidney injury need less dose to recover showing the efficacy of low dose ASV regimen

Conclusion: Our study shows when carefully observe the signs and and symptoms, early hydration, prior treatment with steroids, anti histamines and low dose ASV is non inferior to high dose ASV in effectively treating snake bite patients as ASV neutralizes only unbound, free flowing venom.
\end{abstract}

Keywords: Low dose ASV, Acute kidney injury,

\section{Introduction}

Snake bite is an important problem worldwide, more in tropical countries like India. India is estimated to have the highest snake bite mortality in the world. The true incidence of snake bite is difficult to assess and is often under reported. WHO estimate the number of bites to be 83,000 per annum with 11,000 deaths in India Most of the fatalities are due to the victim not reaching the hospital in time where definite treatment can be administered. In addition community is also not well informed about the occupational risks and simple measures like wearing protective shoes while working in the fields which can prevent the bite. Multiple protocols are being followed for polyvalent anti-snake venom (ASV) administration which has given mixed results in different localities.

\section{Poisonous Snakes}

There are about 236 species of snakes in India, most of which are non-poisonous. However, there are 13 known species that are poisonous and of these four, namely common cobra (Naja naja), Russell's viper (Dabiola russelii), saw-scaled viper (Echis carinatus) and common krait (Bungarus caeruleus) are highly venomous and believed to be responsible for most of the poisonous bites in India.

Early administration of the appropriate dose of potent snake antivenin along with adjuvant treatment, proper care of the wound, correcting electrolyte imbalance, tissue oxygenation, and maintenance of adequate nutrition may help rapid recovery The pathetic story of cobra is its venom capacity and killing capacity. A single bite of cobra contains venom capable of killing 15 to 20 persons. When $13 \mathrm{mg}$ of venom is fatal for an average weight man, it can inject 12 to 20 times such dose and that is why serious cobra bite cases may not reach hospital within one hour. But luckily such cases are very few and may be 2-3\% Krait bites often outnumber 
cobra bites and krait can inject larger than fatal dose in successful bites. Banded Krait bites rarely and it is to be noted that Indian antivenom has no neutralizing effect in this snakebite. Large number of Russell's Viper bite cases come with severe local pain at site of bite with bleeding from the site, with bleeding from gum margins. Sometimes the non-poisonous snakebite causes alarm reactions in a patient like palpitation, restlessness and fear, but this is of no significance on mortality point of view.

\section{Acute kidney injury (AKI)}

Acute kidney injury (AKI) is one of the most significant complications developing due to snake bite. AKI is associated with bites of Russell's Viper, saw-scaled Viper, Puff Adder, Pit Viper and Sea snake. The outcome depends on the type of snake, the area of the body bitten, the amount of venom injected, and the health conditions of the person.

Pathogenesis of AKI in snake bite envenomation
1.Hypotension
2.Intravascular hemolysis
3.DIC
4.Direct nephrotoxicity

One of the main causes of death in snake bite is due to renal failure.

The pathological lesions described in snake bite include

- Mesangial proliferative glomerulonephritis,

- Diffuse proliferative glomerulonephritis,

- Extracapillary proliferative glomerulonephritis,

- Vasculitis

- Tubular necrosis

- Acute diffuse interstitial nephritis (usually associated with tubular necrosis) and cortical necrosis.

- Mesangiolysis is the early appearance of the glomerular lesions

- Fibrin deposition in the peripheral capillary loops and the Bowman's space

- Degeneration, necrosis, and regeneration of tubular epithelial cells have been observed in renal failure following the bite by either haematotoxic or myotoxic snakes

- In haemotoxic snakebite fine granules of haemoglobin can be observed in the proximal tubular cells, and haemoglobin casts are seen in the lumen of necrotic tubules.

- Segmental necrotizing arteritis of the interlobular arteries has been described in Russell's viper bite.

- Segmental thrombophlebitis of the arcuate vein and its tributaries has been reported in both Russell's viper bite and green pit viper bite.

- Deposition of C3 in the wall of afferent and efferent arterioles without any vascular change has been shown in both viper bite and cobra bite patients.

- In cases with recovery there is residual impairment of renal function, and calcification of the renal

Hypotension cortex may be seen by radiography.

Hypotension is one of the important causes of renal failure in snakebite.

- Bleeding either into tissues or externally, and loss of plasma into the bitten extremity can produce hypotension and circulatory collapse. This is caused by venom metallo-proteinases that degrade basement membrane proteins surrounding the vessel wall, leading to loss of integrity.

- Additionally, vasodilatation and increased capillary permeability, both as a result of direct and indirect effects of venom, can aggravate the circulatory disturbances of shock.

- Vipera palestinae venom is thought to cause shock by depression of the medullary vasomotor center.

- Irrespective of the cause, hypotension and circulatory collapse set in motion a chain of hemodynamic disturbances, which are known to culminate in ischaemic ARF.

- Baseline diseases such as ischaemic coronary artery disease, hypertension, diabetes, previous nephropathies, tobacco and alcohol use could be present, making these patients more vulnerable to the effects of snake venom

Prevention and correction of hypotension is a vital component in the management and prevention of renal failure and complications in snake bite. 
Haemodynamic Changes

Haemodynamic changes in snake bite vary among snakes involved.

- In a study of Russell's viper envenomation in canines, initially the cardiac output was decreased,

- Systemic vascular resistance (SVR) and renal vascular resistance (RVR) was increased;

- Renal blood flow (RBF) and the glomerular filtration rate (GFR) were decreased.

- Haemodynamic changes are consistent with the effects of vasoconstrictive mediators.

- The decreased cardiac output is believed to be attributed to the effect of thromboxane A2 resulting in pulmonary artery constriction and decreased blood return to the heart

\section{HOURS AFTER ENVENOMATION}

- Cardiac output was increased;

- SVR was decreased

- RVR was markedly increased;

- $\quad$ RBF and GFR further decreased.

- Haemodynamic alteration at this stage is similar to that of sepsis with prominent effects of NO and PGI2 on systemic circulation and the effect of vasoconstrictive mediators on the kidney.

In most snake envenomations, the glomerular filtration rate and renal blood flow are decreased where renal vascular resistance is increased. There is good correlation between the renal function and the haematologic profile in viper bite.

Additional insults including haemoglobinuria, myoglobinuria, haemorrhage, complement activation and reactive oxygen species play contributing roles in prolonging the duration of decreased GFR and Renal Blood Flow. Renal failure has been observed rarely in patients a few hours after snakebite even without hypotension, haemorrhage, intravascular haemolysis and rhabdomyolysis

Clinical evidence suggests direct nephrotoxicity of the venom.Venomous snakes have enzymes that can directly cause cellular injury. Metalloprotease can cause proteolysis of the extracellular matrix and disrupts cellmatrix and cellular adhesion. Besides the direct injurious effects of the venom, indirect injury can be caused by cytokines and inflammatory mediators induced by both metalloproteases and phospholipase A without haemodynamic changes.

Late (serum sickness-type) reactions develop 1-12 (mean 7) days after treatment. Clinical features include fever, nausea, vomiting, diarrhea, itching, recurrent urticaria, arthralgia, myalgia, lymphadenopathy, immune complex nephritis and, rarely, encephalopathy.

\section{Management of snake bite}

- RIGHT APPROACH

- Reassure

- Immobilize

- Get to Hospital

- Tell about signs of envenomations

Aim of the first aid is to reduce systemic absorption of venom and prevent life-threatening complications.

- No incisions in places where the bite is located, as excessive bleeding and the risk of infection are favored.

- Do not use tourniquets since they hinder blood flow and therefore cause more tissue damage.

- Do not apply ice; it worsens local lesions caused by poison.

- Do not use any chemicals or extracts of plants or animals of any kind, so far none have been proven scientifically effective as treatment

- It is however helpful to note the approximate time of bite as it helps in deciding severity of envenomation.

- Initial victim evaluation include state of airway, breathing, circulatory status, and consciousness.

- Urgent resuscitation is prerequisite in victim with shock, respiratory failure and cardiac arrest. It may include:- 
(A) Oxygen therapy and intravenous access with a large-bore intravenous catheter are a priority. A bolus of normal saline or Ringer's lactate should be given to all patients with suspected envenomation.

(B) Tourniquets though no more recommended, are still used extensively because of lack of awareness. Sudden removal of tourniquet leads to a massive surge of venom. Check for pulse distal to tourniquet. Apply BP cuff with pressure and slowly release it to prevent sudden release of high dose of venom in circulation and its complications

(C) If the victim has brought the snake, try identifying the species carefully, since crotalids can envenomate even when dead.

Considering the high chance of developing kidney impairment after snakebite, the immediate care must include patient's adequate hydration, aiming at a maximum renal protection. However, the most important treatment to prevent AKI is early and specific anti-venom application at the recommended doses.

\section{Dose of ASV;}

- Dose of ASV does not vary with age of victim. ASV should ideally be administered within 4 hours of bite, but is effective even if given within 24 hours.

- ASV neutralizes only unbound, free flowing venom.

- If severe cellulities present asv should be given with in two hours which will prevent compartment syndrome ,tissue necrosis, gangrene , acute kidney injury,

- If the cellulities cross one major joint 5 vials of ASV should be given (two joints 10 ,three 15 immediately).

- The hitch with determining the optimum ASV dose is that the quantity of venom injected at a bite is very variable, depending on the species and size of the snake, the mechanical efficiency of the bite, whether one or two fangs penetrated the skin and whether there were repeated strikes.

- Furthermore, neutralization by antivenin must occur almost immediately after venom enters the circulation to significantly impact on recovery time of the coagulopathy due to envenomation

- The correction of coagulopathy is the most important criteria to continue the ASV treatment.

- After the first dose of bolus ASV, it should be repeated after 6 hours depending on the coagulation profile and may be repeated till the coagulation profile is corrected.

- In snake bite with neurotoxic poisoning after the first dose has been given, another dose may be repeated after $1 \mathrm{hr}$ provided the patients have not improved, or worsened

- the supportive drugs like atropine and neostigmine should be administered according to signs and symptoms to buy time till ASV acts and neutralizes the venom with ventilator support

- As it carries risk of anaphylaxis, epinephrine should thus be kept ready always

- The suggestion of the total requirement of dosages lies between 10 and 30 vials of ASV

- The presently available polyvalent ASV, one effective against bites due to common neurotoxic and haemoto toxic snakes

- It is expensive and scarce-especially in high-risk areas. Although ASV has been used for many years, there is no universal consensus in many centers on the optimal dose and protocol of its administration.

- Theoretically, it would appear that patients with more severe envenomation need higher doses of ASV for effective neutralization of circulating snake venom.

However, many studies have shown that complications and mortality rates are comparable in patients receiving low dose. Thomas et al. showed a higher number of patients requiring ventilator support (7.4\%) and slightly higher number of AKI cases $(22.2 \%)$ with similar mortality rates in low-dose group (7.9 vials of ASV used) when compared to high-dose group (15.3 vials of ASV used $)^{10}$.

In a study conducted by Paul et al., lower number of patients developed AKI in low dose group (18\% vs. $26 \%$ in high dose) and $6 \%$ patients required ventilator support in both groups. Furthermore, mortality rate was slightly lower in low dose compared to high dose $(10 \% \text { vs. } 14 \%)^{11}$.

In a more recent study conducted by Cherian et al., a low dose of ASV was used (6.7 \pm 3.24 vials). Among 54 patients, $12.9 \%$ patients developed AKI, $12.9 \%$ patients required ventilator support and mortality rate was $3.7 \%^{12}$.

Large doses of ASV may not cause any improvement in patients with presynaptic neurotoxicity, which is probably due to the irreversible effects of these toxins (although the clinical significance of presynaptic inhibition is difficult to assess)

.Moreover, due to the high cost and limited availability of ASV, and occasional reports of patients with severe envenomation recovering without the use of ASV, lower dose of ASV may be equally effective and is a considerable option in our Indian setup.

Most of these studies were randomized trials done in India, which has shown little difference in complications between high and low dose ASV usage. 
This study was done in a tertiary hospital in Tamilnadu, where most of the patients are farmers and has the highest number of snakebite cases in this part of India.....

\begin{tabular}{|l|l|l|l|}
\hline & $\mathbf{2 0 1 3}$ & $\mathbf{2 0 1 4}$ & $\mathbf{2 0 1 5}$ \\
\hline Male & 401 & 370 & 847 \\
\hline Female & 48 & 252 & 377 \\
\hline Total & 449 & 622 & 1224 \\
\hline ASV & 6660 & 5765 & 11443 \\
\hline Average ASV used & 14.83 & 9.27 & 9.34 \\
\hline
\end{tabular}

Mortality due to snake bite with AKI being an associated feature

\begin{tabular}{|l|l|l|l|}
\hline & $\mathbf{2 0 1 3}$ & $\mathbf{2 0 1 4}$ & $\mathbf{2 0 1 5}$ \\
\hline Male & 15 & 36 & 23 \\
\hline Female & 9 & 20 & 9 \\
\hline Total & 24 & 56 & 32 \\
\hline Percentage & $5.34 \%$ & $9 \%$ & $2.6 \%$ \\
\hline
\end{tabular}

- Early fluid resuscitation

- Local wound care,

- Low dose ASV and other supportive measures were followed as a protocol in all these patients. .

There is an increasing shortage of ASV in several developing countries and important incentive for a regulated dosing protocol would be to prevent the crisis of ASV availability and supply. In this era of rising medical expenditure and most of the countries facing shortage of ASV, further randomized trials are to be encouraged to determine lower and appropriate doses of ASV in management of snake bite cases.

- The Indian National Snakebite Protocol may also need to be reviewed taking into consideration these newer developments.

\section{Conclusion}

This study has demonstrated that low dose ASV regimen in poisonous snake bites irrespective of the severity along with supportive treatment with emphasis on early fluid resuscitation and other measures as necessary is as efficacious as high dose regime, and has comparable complications including death.

This study was a simple way to analyze these controversies in using lower doses and has successfully shown that using a lower dose has no significant difference in complications and outcome including duration of hospital stay. Whether the routine early use of fresh frozen plasma in patients with features of severe envenomations along with ASV has a preventive role in kidney injury has to be unraveled as intra vascular hemolysis ,bleeding and DIC can be circumvented.

Use of lower dosages could translate into huge savings to the patient and the community

\section{References}

[1]. Kasturiratne A, Wickramsinghe AR, DeSilva N, et al. The global burden of snakebite: A literature analysis and modelling based on regional estimates of envenoming and deaths. PLOS Med. 2008;5:e218

[2]. Sitprija V, Boonpucknavig V. The kidney in tropical snake bite. Clin. Nephrol. 1977; 8: 377-83

[3]. Sitprija V, Boonpucknavig V. Extracapillary proliferative glomerulonephritis in Russell's viper bite. Br. Med. J. 1980; $280: 1417$

[4]. Shastry JCM, Date A, Carman RH, Johny KV. Renal failure following snake bite. Am. J. Trop. Med. Hyg. 1977; 26: 1032-8

[5]. Gundappa RK, Sud K, Kohli HS et al. Snakebite induced acute interstitial nephritis: Report of a rare entity. Ren. Fail. 2002; 24. $369-72$

[6]. Tungthanathanich P, Chaiyabutr N, Sitprija V. Effect of Russell's viper (Vipera russelli siamensis) venom on renal hemodynamics in dogs. Toxicon 1986; 24: 365-71

[7]. Vijeth SR, Dutta TK, Shahapurkar J. Correlation of renal status with hematologic profile in viperine bite. Am. J. Trop. Med. Hyg. 1997; 56: 168-76

[8]. Willinger CC, Thamaree S, Schramek H, Gstraunthaler G, Pfaller W. In vitro nephrotoxicity of Russell's viper venom. Kidney Int 1995; 47:518-28

[9]. Isbister GK, Duffull SB, Brown SG, ASP Investigators. Failure of antivenom to improve recovery in Australian snakebite coagulopathy. QJM. 2009;102:563-8.

[10]. Thomas PP, Jacob J. Randomised trial of antivenom in snake envenomation with prolonged clotting time.Br Med J (Clin Res Ed) $1985 ; 291: 177-8$

[11]. Paul V, Pratibha S, Prahlad KA, Earali J, Francis S, Lewis F. High-dose anti-snake venom versus low-dose anti-snake venom in the treatment of poisonous snake bites-a critical study. J Assoc Physicians India.2004;52:14-7

[12]. Cherian AM, Girish TS, Jagannati M, Lakshmi M. High or low-A trial of low dose anti snake venom in the treatment of poisonous snakebites. J Assoc Physicians India. 2013;61:387-9, 396 